\title{
On the Virasoro Structure of Symmetry Algebras of Nonlinear Partial Differential Equations
}

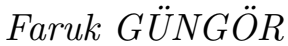

Department of Mathematics, Faculty of Science and Letters, Istanbul Technical University, 34469, Istanbul, Turkey

E-mail: gungorf@itu.edu.tr

URL: http://www.mat.itu.edu.tr/gungor/

Received November 30, 2005, in final form January 20, 2006; Published online January 30, 2006

Original article is available at http://www.emis.de/journals/SIGMA/2006/Paper014/

\begin{abstract}
We discuss Lie algebras of the Lie symmetry groups of two generically nonintegrable equations in one temporal and two space dimensions arising in different contexts. The first is a generalization of the KP equation and contains 9 arbitrary functions of one and two arguments. The second one is a system of PDEs that depend on some physical parameters. We require that these PDEs are invariant under a Kac-Moody-Virasoro algebra. This leads to several limitations on the coefficients (either functions or parameters) under which equations are prime candidates for being integrable.
\end{abstract}

Key words: Kadomtsev-Petviashvili and Davey-Stewartson equations; symmetry group; Virasoro algebra

2000 Mathematics Subject Classification: 35A30; 35Q53; 35Q55; 35Q58

\section{Introduction}

It is well known that a number of physically significant integrable partial differential equations in 2+1-dimensions typically have infinite-dimensional symmetry algebras with a specific KacMoody-Virasoro (KMV) structure. Among them one can cite the Kadomtsev-Petviashvili (KP) equation [1, 2], modified KP, cylindrical KP equation [3], all equations of the KP hierarchy [4], Davey-Stewartson (DS) system [5] and three-wave resonant interaction equations [6]. On the other hand, it should be mentioned that there are evolution type equations which are integrable, but do admit infinite-dimensional symmetry algebras without a KMV structure. For instance, the breaking soliton equation and Zakharov-Strachan equation [7] do not allow a KMV type symmetry algebra while they are integrable. This observation shows that the existence of a KMV symmetry algebra is not a necessary condition for integrability for a nonlinear evolution equation in $2+1$ dimensions. Nevertheless, it is our firm belief that identifying equations with KMV symmetry algebra can serve us to provide those subclasses which are candidates for integrability. Of course, integrable ones must be further singled out by checking them for integrability in any sense of the word. To mention a few, one can perform singularity analysis for establishing Painlevé property or proceed to study higher symmetries or look for a Lax pair.

In this paper we shall concentrate on generalizations of two integrable equations in two space dimensions. One is the generalized KP (GKP) equation (prototype of a 2+1-dimensional integrable equation) and the other is generalized DS (GDS) equation. For both systems we shall determine the cases when the equations admit an infinite-dimensional symmetry group the Lie algebra of which has a Virasoro structure. It will be shown that how this requirement will impose restrictions on the coefficients. 
First we consider the GKP equation

$$
\begin{aligned}
\left(u_{t}+p(t) u u_{x}+q(t) u_{x x x}\right)_{x}+\sigma(y, t) u_{y y}+a(y, t) u_{y} & \\
& +b(y, t) u_{x y}+c(y, t) u_{x x}+e(y, t) u_{x}+f(y, t) u+h(y, t)=0 .
\end{aligned}
$$

We assume that in some neighbourhood we have

$$
p(t) \neq 0, \quad q(t) \neq 0, \quad \sigma(y, t) \neq 0 .
$$

The other functions in (1) are arbitrary.

Second, we consider the system

$$
\begin{aligned}
& i \psi_{t}+\delta \psi_{x x}+\psi_{y y}=\chi|\psi|^{2} \psi+\gamma\left(w_{x}+\phi_{y}\right) \psi, \\
& w_{x x}+n \phi_{x y}+m_{2} w_{y y}=\left(|\psi|^{2}\right)_{x}, \\
& n w_{x y}+\lambda \phi_{x x}+m_{1} \phi_{y y}=\left(|\psi|^{2}\right)_{y},
\end{aligned}
$$

with the condition $(\lambda-1)\left(m_{1}-m_{2}\right)=n^{2}$. Here $\psi(t, x, y)$ is a complex function, $w(t, x, y)$ and $\phi(t, x, y)$ are real functions and $\delta, n, m_{1}, m_{2}, \lambda, \chi, \gamma$ are real constants. This system of nonlinear partial differential equations in $2+1$ dimensions arises as a model of wave propagation in a bulk medium composed of an elastic material with couple stresses [8].

\section{Kac-Moody-Virasoro (KMV) algebras and their subalgebras}

We recall that a KMV algebra is an infinite-dimensional Lie algebra with a basis $[9,10]$

$$
\left\{L_{m}, T_{m}^{a}, C, K\right\}, \quad 1 \leq a \leq N, \quad m \in \mathbb{Z},
$$

satisfying the commutation relations

$$
\begin{aligned}
& {\left[L_{m}, L_{n}\right]=(m-n) L_{m+n}+\frac{1}{12} m\left(m^{2}-1\right) \delta_{m,-n} C,} \\
& {\left[T_{m}^{a}, T_{m}^{b}\right]=f^{a b c} T_{m+n}^{c}+m \delta_{a, b} \delta_{m,-n} K, \quad\left[L_{m}, T_{n}^{a}\right]=-n T_{m+n}^{a},} \\
& {\left[C, L_{m}\right]=\left[C, T_{m}^{a}\right]=\left[K, L_{m}\right]=\left[K, T_{m}^{a}\right]=[K, C]=0,}
\end{aligned}
$$

where $f^{a b c}$ are the structure constants of some finite-dimensional real or complex simple Lie algebra $A$. The elements $\left\{T_{m}^{a}, K\right\}$ form the basis of a Kac-Moody algebra, $\left\{L_{m}, C\right\}$ form the Virasoro algebra, $K$ and $C$ are central elements, namely they commute with all other elements and with each other. From (4), it is seen that the Kac-Moody algebra is an ideal in the entire structure. KMV algebras seem to arise in many branches of theoretical physics and in the theory of completely integrable systems.

A simple realization of the algebra (3) is obtained by introducing a scalar parameter $\lambda$, a finite-dimensional Lie algebra $A$ with basis $\left\{X^{1}, \ldots, X^{N}\right\}$ and commutation relations

$$
\left[X^{a}, X^{b}\right]=f^{a b c} X^{c}, \quad a, b, c=1,2, \ldots, N .
$$

We put

$$
L_{m}=-\lambda^{m+1} \partial_{\lambda}, \quad T_{m}^{a}=X^{a} \lambda^{m}, \quad C=0, \quad K=0
$$

and see that the commutation relations (4) are satisfied. As the central elements $C$ and $K$ are represented trivially in (5), we actually have a representation of an affine loop algebra. For example, the set of (complex) vector fields on the unit circle

$$
L_{m}=-i e^{i m t} \partial_{t}
$$

satisfies

$$
\left[L_{m}, L_{n}\right]=(m-n) L_{m+n},
$$

and therefore realizes a Virasoro algebra without a center. 


\subsection{The symmetry algebra of CGKP equation}

With the introduction of allowed transformations [11] (point transformations taking equation into one with different coefficients) we can transform (1) into some canonical form

$$
\begin{gathered}
\left(u_{t}+u u_{x}+u_{x x x}\right)_{x}+\varepsilon u_{y y}+a(y, t) u_{y}+b(y, t) u_{x y} \\
+c(y, t) u_{x x}+f(y, t) u=0, \quad \varepsilon= \pm 1 .
\end{gathered}
$$

We call (6) canonical GKP equation and abbreviate CGKP equation.

If we restrict ourselves to Lie point symmetries of equation (6), the Lie algebra of the symmetry group is represented by vector fields of the form

$$
\boldsymbol{V}=\xi \partial_{x}+\eta \partial_{y}+\tau \partial_{t}+\phi \partial_{u}
$$

where $\xi, \eta, \tau$ and $\phi$ are functions of $x, y, t$ and $u$. The method for the determination of the coefficients of the vector field $\boldsymbol{V}$ is algorithmic [12]. Applying this to (6) gives an overdetermined set of linear partial differential equations for the coefficients $\xi, \eta, \tau$ and $\phi$ in equation (7). Solving this system we find that the vector field (7) should have the form

$$
\begin{aligned}
\boldsymbol{V}= & \tau(t) \partial_{t}+\left(\frac{1}{3} \dot{\tau} x+\xi_{0}(y, t)\right) \partial_{x} \\
& +\left(\frac{2}{3} \dot{\tau} y+\eta_{0}(t)\right) \partial_{y}+\left(-\frac{2}{3} \dot{\tau} u+\frac{1}{3} \ddot{\tau} x+S(y, t)\right) \partial_{u}
\end{aligned}
$$

where

$$
S(y, t)=-\tau c_{t}-\left(\frac{2}{3} \dot{\tau} y+\eta_{0}\right) c_{y}+\xi_{0, t}+b \xi_{0, y}-\frac{2}{3} c \dot{\tau},
$$

and $\tau(t), \eta(t)$ and $\xi_{0}(y, t)$ satisfy

$$
\begin{aligned}
& 3 \tau a_{t}+\left(2 \dot{\tau} y+3 \eta_{0}\right) a_{y}+2 a \dot{\tau}=0, \\
& -3 \dot{\eta}_{0}-2 y \ddot{\tau}+3 \tau b_{t}+\left(2 \dot{\tau} y+3 \eta_{0}\right) b_{y}+b \dot{\tau}-6 \varepsilon \xi_{0, y}=0, \\
& \ddot{\tau}+3 a \xi_{0, y}+3 \varepsilon \xi_{0, y y}=0, \\
& f \ddot{\tau}=0, \\
& 4 f \dot{\tau}+3 f_{t} \tau+f_{y}\left(2 \dot{\tau} y+3 \eta_{0}\right)=0, \\
& \dddot{\tau}+3 f S+3 a S_{y}+3 \varepsilon S_{y y}=0,
\end{aligned}
$$

where $S(y, t)$ of equation (9) should be substituted into equation (10).

We shall determine the conditions on the functions $a, b, c$ and $f$ that allow the symmetry algebra to be infinite-dimensional with an additional KMV structure.

\subsection{Virasoro symmetries of the CGKP equation}

The canonical generalized KP equation (6) will be invariant under a transformation group, the Lie algebra of which is isomorphic to a Virasoro algebra if the function $\tau$ in (8) remains free. Omitting details we summarize the main results as a theorem:

Theorem 1. The canonical generalized KP equation (6) allows the Virasoro algebra as a symmetry algebra if and only if the coefficients satisfy

$$
a=f=0, \quad b=b(t), \quad c=c_{0}(t)+c_{1}(t) y .
$$


Theorem 2. The canonical generalized KP equation

$$
\left(u_{t}+u u_{x}+u_{x x x}\right)_{x}+\varepsilon u_{y y}+b(t) u_{x y}+\left[c_{0}(t)+c_{1}(t) y\right] u_{x x}=0
$$

with $\varepsilon= \pm 1$, and $b(t), c_{0}(t)$ and $c_{1}(t)$ arbitrary smooth functions is invariant under an inf initedimensional Lie point symmetry group. Its Lie algebra has a Kac-Moody-Virasoro structure. It is realized by vector fields of the form

$$
\boldsymbol{V}=T(\tau)+X(\xi)+Y(\eta)
$$

where $\tau(t), \xi(t)$ and $\eta(t)$ are arbitrary smooth functions of time and we have

$$
\begin{aligned}
T(\tau)= & \tau(t) \partial_{t}+\frac{1}{6}\left[3 \varepsilon \dot{b} y \tau+(2 x+\varepsilon b y) \dot{\tau}-\varepsilon \ddot{\tau} y^{2}\right] \partial_{x}+\frac{2}{3} \dot{\tau} \partial_{y} \\
& +\frac{1}{6}\left\{\left[-6 \dot{c}_{0}+3 \varepsilon b \dot{b}+\left(-6 \dot{c}_{1}+3 \varepsilon \ddot{b}\right) y\right] \tau+\left[-4 u+\varepsilon b^{2}-4 c_{0}+4\left(\varepsilon \dot{b}-2 c_{1}\right) y\right] \dot{\tau}\right. \\
& \left.+(2 x-\varepsilon b y) \ddot{\tau}-\varepsilon y^{2} \dddot{\tau}\right\} \partial_{u}, \\
X(\xi)= & \xi(t) \partial_{x}+\dot{\xi}(t) \partial_{u}, \\
Y(\eta)= & \eta(t) \partial_{y}-\frac{\varepsilon}{2} \dot{\eta}(t) y \partial_{x}-\frac{1}{2}\left[2 c_{1} \eta+\varepsilon b \dot{\eta}+\varepsilon y \ddot{\eta}\right] \partial_{u} .
\end{aligned}
$$

Remark 1. The transformation

$$
\begin{aligned}
& u(x, y, t)=\tilde{u}(\tilde{x}, \tilde{y}, \tilde{t})+\left(\frac{\varepsilon}{2} \dot{b}-c_{1}\right) y-c_{0}+\frac{\varepsilon}{4} b^{2}, \\
& \tilde{x}=x-\frac{\varepsilon b}{2} y, \quad \tilde{y}=y, \quad \tilde{t}=t
\end{aligned}
$$

takes equation (12) into the KP equation itself, i.e. into equation (12) with $b=c_{0}=c_{1}=0$. The transformation (17) also transforms the Lie algebra (13)-(16) into the symmetry algebra [1] of the KP equation.

Theorem 3. The GKP equation (1) is invariant under a Lie point symmetry group, the Lie algebra of which contains a Virasoro algebra as a subalgebra, if and only if it can be transformed into the KP equation itself by a point transformation.

In summary, we have identified all cases when the generalized KP equation has an infinitedimensional symmetry group whose Lie algebra has a Virasoro structure. For a complete analysis and some implications of these results we refer the reader to [11].

A natural question to ask is what can one do with the integrable CGKP (12)? The tools of soliton theory such as the inverse spectral transform and Bäcklund transformations are at our disposal to obtain multisoliton and other physically important solutions of this equation.

A general class of fourth order scalar partial differential equations, invariant under the group of local point transformations of the KP equation has been constructed in [13]. We note that a recent paper [14] has been devoted to the construction of equations of arbitrary order invariant under the KP symmetry group. They searched for an autonomous higher order KP family

$$
\left(u_{t}+\frac{3}{2} u u_{x}+u_{x x x}\right)_{x}+\frac{3}{4} \delta u_{y y}+F(u)=0,
$$

where $F(u)$ is a differential function of $u$ and its any order derivatives of $x, y, t$ variables with no space-time dependence explicitly occurring in it, which possesses the same KMV symmetry algebra as the standard KP equation. The idea used in [14] is similar to the one we used above. The KP family is required to be left invariant under the KP symmetry group. The Virasoro 
algebra will be present in the entire symmetry algebra as a subalgebra whenever symmetry equations (obtained from splitting of the linearized equation) can be solved identically with the function $\tau(t)$ ( $t$-coefficient of the vector field) arbitrary. This implies that the equation is invariant under an arbitrary reparametrization of time. These type of equations will include the most probable candidates for integrability (integrable models).

In another paper [15], an attempt was made to determine whether there can exist any possible variable coefficient extensions of the $\mathrm{KP}$ equation

$$
\left(u_{t}+f(x, y, t) u u_{x}+g(x, y, t) u_{x x x}\right)_{x}+h(x, y, t) u_{y y}=0,
$$

such that it can not be transformed to the standard KP equation by allowed transformations, but still can have an infinite-dimensional symmetry group.

We now turn to the system (2). For our purposes we find it more convenient to study the differentiated form of it with the first complex equation separated into real and imaginary components via $\psi=u+i v$

$$
\begin{aligned}
& u_{t}+\delta v_{x x}+v_{y y}=\chi v\left(u^{2}+v^{2}\right)+\gamma v(w+\phi), \\
& -v_{t}+\delta u_{x x}+u_{y y}=\chi u\left(u^{2}+v^{2}\right)+\gamma u(w+\phi), \\
& w_{x x}+n \phi_{x x}+m_{2} w_{y y}=2\left(u_{x}^{2}+u u_{x x}+v_{x}^{2}+v v_{x x}\right), \\
& n w_{y y}+\lambda \phi_{x x}+m_{1} \phi_{y y}=2\left(u_{y}^{2}+u u_{y y}+v_{y}^{2}+v v_{y y}\right) .
\end{aligned}
$$

Again, we represent a general element of the symmetry algebra by a vector field of the form

$$
\boldsymbol{V}=\tau \partial_{t}+\xi \partial_{x}+\eta \partial_{y}+\varphi_{1} \partial_{u}+\varphi_{2} \partial_{v}+\varphi_{3} \partial_{w}+\varphi_{4} \partial_{\phi}
$$

where the coefficients $\tau, \xi, \eta, \varphi_{i}, i=1,2,3,4$ are functions of $t, x, y, u, v, w, \phi$. Applying the symmetry procedure we find

$$
\boldsymbol{V}=T(f)+X(g)+Y(h)+W(m),
$$

where

$$
\begin{aligned}
T(f)= & f(t) \partial_{t}+\frac{1}{2} f^{\prime}(t)\left(x \partial_{x}+y \partial_{y}-u \partial_{u}-v \partial_{v}-2 w \partial_{w}-2 \phi \partial_{\phi}\right) \\
& -\frac{\left(x^{2}+\delta y^{2}\right)}{8 \delta}\left[f^{\prime \prime}(t)\left(v \partial_{u}-u \partial_{v}\right)+\frac{f^{\prime \prime \prime}(t)}{2 \gamma}\left(\partial_{w}+\partial_{\phi}\right)\right], \\
X(g)= & g(t) \partial_{x}-\frac{x}{2 \delta}\left[g^{\prime}(t)\left(v \partial_{u}-u \partial_{v}\right)+\frac{g^{\prime \prime}(t)}{2 \gamma}\left(\partial_{w}+\partial_{\phi}\right)\right], \\
Y(h)= & h(t) \partial_{y}-\frac{y}{2}\left[h^{\prime}(t)\left(v \partial_{u}-u \partial_{v}\right)+\frac{h^{\prime \prime}(t)}{2 \gamma}\left(\partial_{w}+\partial_{\phi}\right)\right], \\
W(m)= & m(t)\left(v \partial_{u}-u \partial_{v}\right)+\frac{m^{\prime}(t)}{2 \gamma}\left(\partial_{w}+\partial_{\phi}\right) .
\end{aligned}
$$

The functions $g(t), h(t)$, and $m(t)$ are arbitrary functions of class $C^{\infty}(I), I \subseteq \mathbb{R}$. The function $f(t)$ is arbitrary if

$$
m_{2} \delta+n+1=0, \quad m_{1} \delta+n \delta+\lambda=0,
$$

otherwise $f(t)=c_{2} t^{2}+c_{1} t+c_{0}$. We focus on the case when $f(t)$ is allowed to be arbitrary for which the symmetry algebra realized by the vector fields (19) and (20) is then infinite-dimensional. The commutation relations for the GDS algebra are as follows:

$$
\left[T\left(f_{1}\right), T\left(f_{2}\right)\right]=T\left(f_{1} f_{2}^{\prime}-f_{1}^{\prime} f_{2}\right), \quad[T(f), X(g)]=X\left(f g^{\prime}-\frac{1}{2} f^{\prime} g\right),
$$




$$
\begin{aligned}
& {[T(f), Y(h)]=Y\left(f h^{\prime}-\frac{1}{2} f^{\prime} h\right), \quad[T(f), W(m)]=W\left(f m^{\prime}\right),} \\
& {\left[X\left(g_{1}\right), X\left(g_{2}\right)\right]=-\frac{1}{2 \delta} W\left(g_{1} g_{2}^{\prime}-g_{1}^{\prime} g_{2}\right), \quad\left[Y\left(h_{1}\right), Y\left(h_{2}\right)\right]=-\frac{1}{2} W\left(h_{1} h_{2}^{\prime}-h_{1}^{\prime} h_{2}\right),} \\
& {[X(g), Y(h)]=[X(g), W(m)]=[Y(h), W(m)]=\left[W\left(m_{1}\right), W\left(m_{2}\right)\right]=0 .}
\end{aligned}
$$

They characterize the commutation relations of a centerless KMV algebra which is identified as the semi-direct sum (actually a Levi decomposition) $L=S \in N$, where $S=\{T(f)\}$ is a simple infinite dimensional Lie algebra and $N=\{X(g), Y(h), W(m)\}$ is a nilpotent ideal (nilradical).

More interestingly, the GDS (18) system has a Lie symmetry algebra $L$ isomorphic to that of the DS symmetry algebra (the symmetry algebra of the integrable DS equations) [5]

$$
\begin{aligned}
& i \psi_{t}+\delta_{1} \psi_{x x}+\psi_{y y}=\delta_{2}|\psi|^{2} \psi+w \psi, \\
& \varepsilon_{1} w_{x x}+w_{y y}=\varepsilon_{2}\left(|\psi|^{2}\right)_{y y},
\end{aligned}
$$

with $\delta_{1}= \pm 1, \delta_{2}= \pm 1$ and $\delta_{1}+\varepsilon_{1}=0$.

We have the following (full details can be found in [16]):

Theorem 4. The system (18) is invariant under an inf inite-dimensional Lie point symmetry group, the Lie algebra of which has a Kac-Moody-Virasoro structure isomorphic to the DS algebra if and only if the conditions (21) hold.

Presence of isomorphism suggests to look for a point transformation taking symmetry algebras into each other. Hence we can possibly expect that the corresponding equations are transformed into each other under such a transformation. Let us mention that there is indeed a transformation $q=w+\phi-|\psi|^{2}$ reducing the GDS symmetry algebra to the DS algebra. However, this transformation does not appear to be one-to-one because both systems do not have the same number of dependent variables.

\section{Conclusions}

In this paper, we studied group theoretical properties of two classes of nonlinear evolution equations in (2+1)-dimensions, namely generalized KP and DS equations. In particular, we demanded these equations to be invariant under the infinite-dimensional Lie groups of local point transformations with additional KMV structure. We showed that for the generalized KP equation (1), this is possible only if the coefficients satisfy (11). This is the only case when the original equation can be transformed to the KP equation itself. For the generalized DS equations, we showed that the KMV symmetry algebra can be present only if the conditions (21), under which the symmetry algebra is isomorphic to that of the integrable DS equations, hold.

Despite the usefulness of existence of KMV symmetry algebras for equations in two space dimensions, many fundamental questions still remain open. The precise relationship between symmetries and integrability is still far from being completely understood. As was noted in [7], not all integrable equations admit KMV symmetry algebras. On the other hand, all known non-integrable equations are invariant under finite or infinite-dimensional Lie point transformation groups without KMV structure. These facts demonstrate that a fuller understanding of the absence of KMV structure in an infinite-dimensional symmetry algebra is essential in this context. If the existence of a KMV symmetry algebra alone can not be a necessary condition for integrability for a nonlinear evolution equation in $2+1$ dimensions, so what conditions must be added to ensure integrability? 


\section{Acknowledgements}

The author is grateful to the referees for useful comments and acknowledges the financial support from Istanbul Technical University (ITU).

[1] David D., Kamran N., Levi D., Winternitz P., Subalgebras of loop algebras and symmetries of the Kadomtsev-Petviashvili equation, Phys. Rev. Let., 1985, V.55, N 20, 2111-2113.

[2] David D., Kamran N., Levi D., Winternitz P., Symmetry reduction for the Kadomtsev-Petviashvili equation using a loop algebra, J. Math. Phys., 1986, V.27, N 5, 1225-1237.

[3] Levi D., Winternitz P., The cylindrical Kadomtsev-Petviashvili equation, its Kac-Moody-Virasoro algebra and relation to the KP equation, Phys. Lett. A, 1988, V.129, 165-167.

[4] Orlov A.Yu., Winternitz P., Algebra of pseudodifferential operators and symmetries of equations in the Kadomtsev-Petviashvili hierarchy, J. Math. Phys., 1997, V.38, 4644-4674.

[5] Champagne B., Winternitz P., On the infinite dimensional symmetry group of the Davey-Stewartson equation, J. Math. Phys., 1988, V.29, 1-8.

[6] Martina L., Winternitz P., Analysis and applications of the symmetry group of the multidimensional threewave resonant interaction problem, Ann. Phys., 1989, V.196, 231-277.

[7] Senthil Velan M., Lakshmanan M., Lie symmetries, Kac-Moody-Virasoro algebras and integrability of certain (2+1)-dimensional nonlinear evolution equations, J. Nonlinear Math. Phys., 1998, V.5, 190-211; math-ph/9804017.

[8] Babaoglu C., Erbay S., Two-dimensional wave packets in an elastic solid with couple stresses, Internat. J. Non-Linear Mech., 2004, V.39, 941-949.

[9] Winternitz P., Kac-Moody-Virasoro symmetries of integrable nonlinear partial differential equations, in Symmetries and Nonlinear Phenomena, Editors D. Levi and P. Winternitz, Singapore, World Scientific, 1988, 358-375.

[10] Winternitz P., Group theory and exact solutions of partially integrable differential systems, in Partially Integrable Evolution Equations in Physics (1989, Les Houches), Editors R. Conte and N. Boccara, NATO Adv. Sci. Inst. Ser. C Math. Phys. Sci., V.310, Dordrecht, Kluwer Acad. Publ., 1990, 515-567.

[11] Güngör F., Winternitz P., Generalized Kadomtsev-Petviashvili equation with an infinite dimensional symmetry algebra, J. Math. Anal. Appl., 2002, V.276, 314-328; nlin.SI/0109009.

[12] Olver P.J., Applications of Lie groups to differential equations, New York, Springer, 1991.

[13] David D., Levi D., Winternitz P., Equations invariant under the symmetry group of the KadomtsevPetviashvili equation, Phys. Lett. A, 1988, V.129, 161-164.

[14] Lou S.Y., Tang X.Y., Equations of arbitrary order invariant under the Kadomtsev-Petviashvili symmetry group, J. Math. Phys., 2004, V.43, 1020-1030.

[15] Güngör F., Winternitz P., Equivalence classes and symmetries of the variable coefficient KP equation, Nonlinear Dynam., 2004, V.35, 381-396.

[16] Güngör F., Aykanat Ö., The generalized Davey-Stewartson equations, its Kac-Moody-Virasoro symmetry algebra and relation to Davey-Stewartson equations, J. Math. Phys., 2006, to appear. 